\title{
Frictional Force Determination of New Implements with Synchronous Belt for Hauling up Purse Seine Net
}

\author{
Cao Guangbin*1 and Sadami Yada ${ }^{* 2}$ \\ ${ }^{*}$ Heilongjian Fisheries Institute, Fisheries Science Research Academy of China, \\ 43 Shongfa Street, Daoli, Harbin 150070, China \\ ${ }^{*}$ Department of Marine Science and Technology, Tokyo University of Fisheries, \\ Konan, Minato, Tokyo 108, Japan
}

(Received April 9, 1996)

\begin{abstract}
The frictional force acting on the vertical synchronous belts used in hauling up the net or rope and the main factors affecting the frictional forces were determined through experimental and theoretical analysis. The frictional force was calculated by using Euler's equation. It was shown that the average frictional coefficient between belt and net was 0.78 and that between belt and rope was 0.70 . When the net or rope was wound out by belts along a spiral line, the frictional force was found to decrease with an increase in the spiral angle. At a constant velocity, the speed of moving up the net or rope had no effect upon the frictional force. On the other hand, a non-uniform velocity of moving reduced the actual frictional force.
\end{abstract}

Key words: frictional force, net hauler, hauling drum, synchronous belt, fishing net, fishing rope, fishing machine

Prediction of the frictional force is necessary when determining the pulling force at the stage of designing fishing implements such as rope winches, ${ }^{1}$ net hauling powered blocks ${ }^{2}$ and net haulers. ${ }^{3)}$ The pulling force changes with variations in the working conditions in accordance with different frictional performance of different net haulers. Due to the mechanical limitations of the presently used net haulers, a part of the fishing net is wound on a circular part at a very small contact angle. The frictional force is limited and sometimes the pulling force is not large enough to haul the fishing net. ${ }^{2)}$ The key to improving the performance of net haulers is to increase the frictional contact angle and decrease the pulling force. The larger the frictional contact angle, the smaller the pulling force, and hence the larger the load that can be hauled up by the net hauler for the same pulling force. Therefore, the authors designed a new kind of net hauler with vertical synchronous belts. The new mechanism for the net hauler is based on a spiral motion, in which the net is wound onto a drum made of six pairs of synchronous belts surrounding the drum; these synchronous belts are used to move up the fishing net. By winding the net three to four times around the drum, the frictional contact angle is enlarged up to $3 \pi$. Thus, the frictional force is greatly increased and the hauling performance improved. ${ }^{4)}$ This paper also reports on frictional experiments conducted on the synchronous belts and the net or rope, frictional force determinations and some deisgn parameters. Synchronous belts are widely utilized in many machines ${ }^{5}$ as transmission elements, and many studies have been carried out on their effectiveness. ${ }^{(-10)}$ However, few studies have been made on the synchronous belts used as load transporting elements and previous papers have not been concerned with the synchronous belts used to haul the nets or ropes in fishing operations.
In order to derive the technical design parameters for calculating the frictional force and determining the pulling force of the net hauler with synchronous belts and to design new mechanism implements, one of the six pairs of synchronous belts was used for frictional experiments to analyze the effects of each factor on frictional force.

\section{Experimental Implements and Methods}

\section{Experimental Apparatus and Materials}

The new net hauler with vertical synchronous belts is shown in Fig. 1. The whole body of the drum is installed on the deck with the baseplate 1 . The driving shaft 2 makes the supporting structure 6 revolve with the whole drum through the gears 3 and 5 . As the drum revolves, the bevel gear 10 turns, which meshes with the fixed gear 9 . Power is transferred to the driving belt pulley 13 by the bevel gears 11 and 12 , so it is possible for the synchronous belt 7 to move up while the drum revolves.

When it is used in purse seine fishing operations, at first the head parts of the sinker line and float line are connected to a guiding rope, which has initially been wound three to four times around the drum, then the implements are put into operation. During the net hauling operation, the guiding rope assists the net to pass through a net binding groove, and the bound net bundle is guided onto the drum, which has six pairs of the synchronous belt 7 wound around it. As the drum rotates with the net, the net is carried up by the synchronous belt 7 . In normal operation, the wound net is stationary on the belts of the drum. After turning three to four times in a spiral motion on the drum, the net is transported to a certain height, and then is guided to a net outgoing groove. Because the net is transported in a spiral motion, and the pitch of the spiral is slightly larger than the width of the net bundle, the net can be 


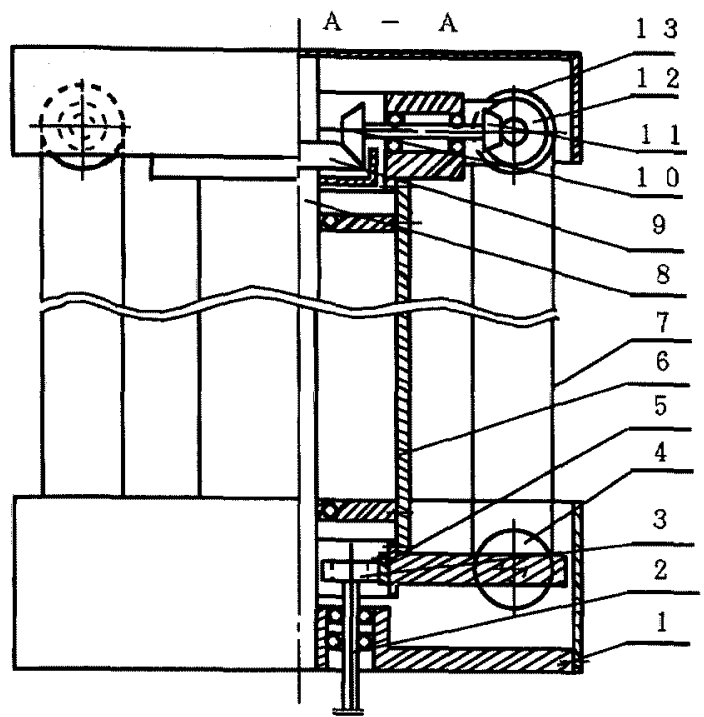

a: section view

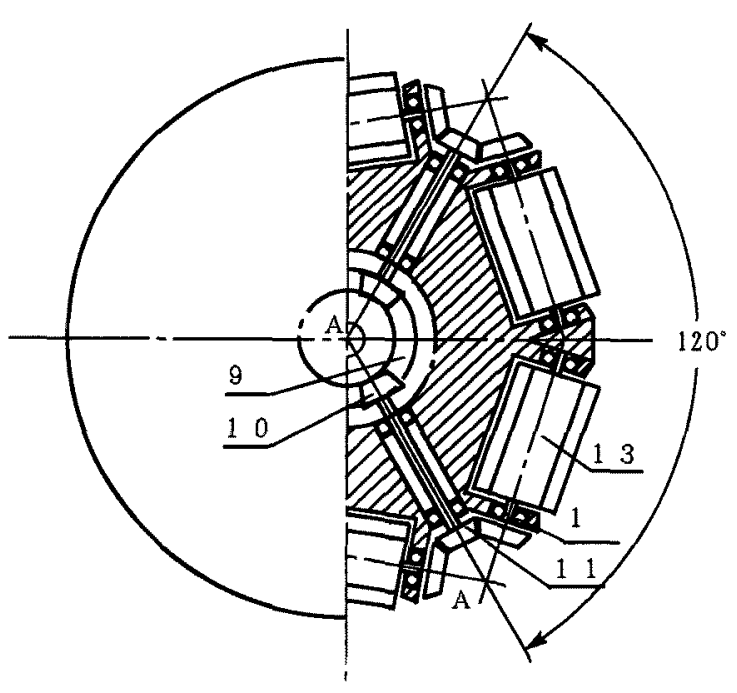

b: plane view

Fig. 1. New mechanism of transmission draft of net hauling drum. 1: baseplate, 2: driving shaft, 3 : driving gear, 4 : driven belt pulley, 5: driven gear, 6 : supporting structure, $7:$ synchronous belt, 8 : fixed main shaft, 9: fixed gear, 10,11 and 12: bevel gear, 13: driven belt pulley.

wound three to four times on the drum without any interference among the turns. By doing so, the contact angle between the net and drum is enlarged and the pulling force increased greatly. Thus, the new net hauling mechanism has the advantages of large pulling force, high hauling velocity and easy net handling. ${ }^{4}$

The experiments investigated the frictional force of the new mechanism. For each of the six pairs of synchronous belts, the friction between the synchronous belt and the net or rope had the same working conditions. The experiments were carried out with the implements shown in Fig.
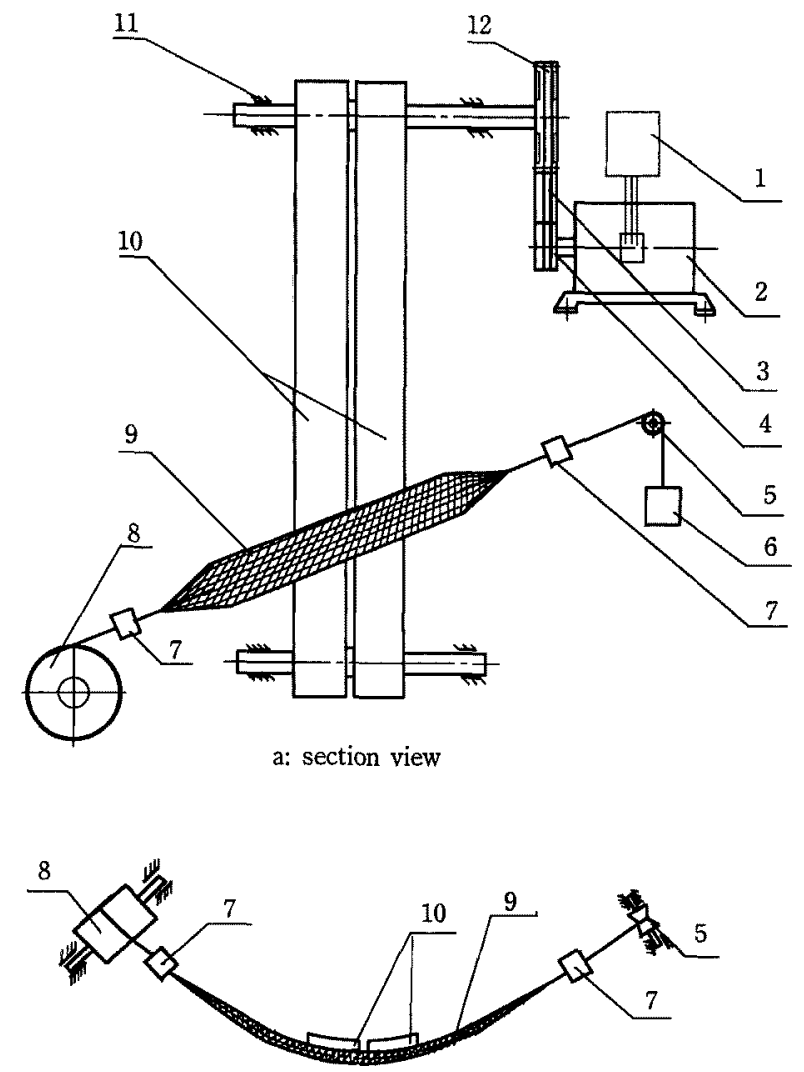

b: plane view

Fig. 2. Operation diagram of frictional experiments.

1: inverter, 2: motor, 3: V-belt, 4; driving pulley, 5: roller, 6: weight, 7: tension meter cell, 8: which, 9: net or rope, 10: synchronous belt, 11: bearing, 12: driven pulley.

2 , and were designed to reproduce the working conditions of the new vertical synchronous belts. The pulling and hauling load were measured with tension meter cells and the data were taken with a NEC-RT3104 recorder. The control apparatus (Freqral-Z200 inverter) was used for measuring the hauling velocity. The transporting element of the implements was a pair of simultaneously moving synchronous belts (JIS K6372 General $1200 \mathrm{XH} 400$ ) and the frictional experiments were made for a rope (polyethylene four-strand twisted rope of $18 \mathrm{~mm}$ ) and a piece of net (weaver's knotted netting, 100 mesh size with a nylon six yarns twisted line of $0.5 \mathrm{~mm}$ ). The synchronous belts were used to move the net or rope up. The net was shaped in such a way so as to simulate the hauling conditions of the real net.

\section{Experimental Methods}

The experiments were carried out according to the following steps.

Determination of Frictional Force at Belt Velocity of Zero As shown in Figs. 2 and 3, when the spiral angle $\beta$ was determined, on the outgoing end of the net or rope pulling force $P_{2}$ was applied by a weight 6 , on the incoming end load $P_{1}$ was gradually applied by the winch 8 . When the 


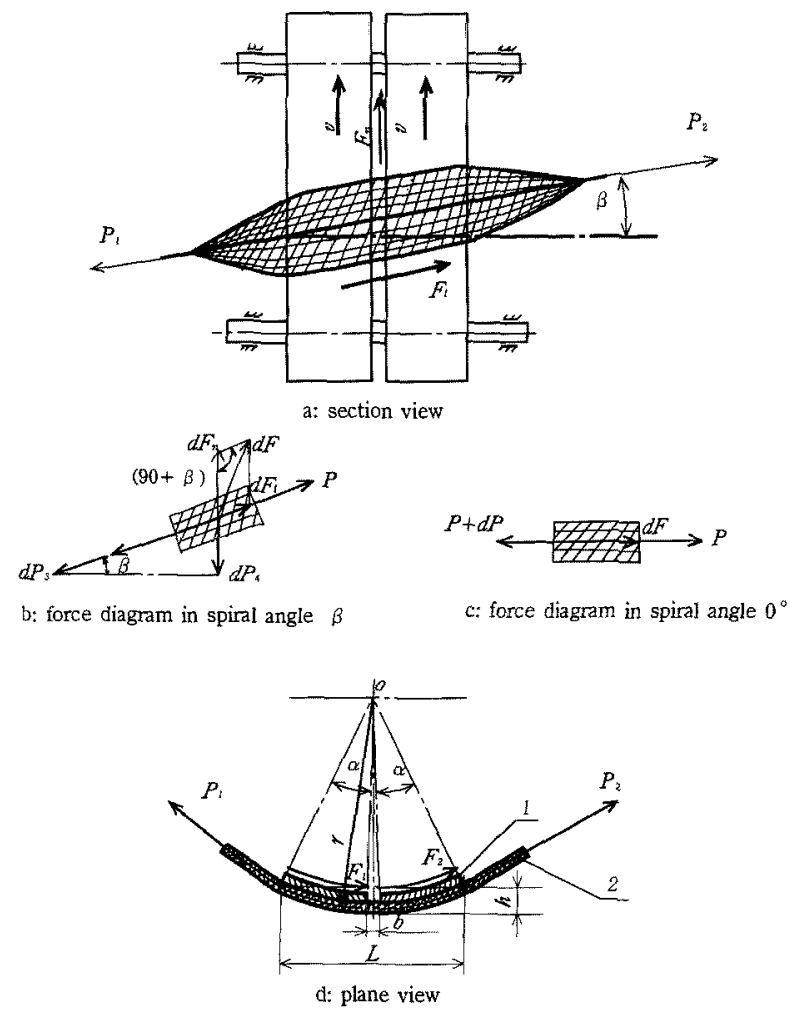

Fig. 3. Force diagram of synchronous belt frictional experiments.

1: synchronous belt, 2: net or rope, $\alpha$ : contact angle $(\beta=0), r$ radius of circular arch, $\beta$ : spiral angle, $b$ : distance between belt, $h$ : arch height of net or rope, $L:$ arch length of net or rope, $P$ : tension in any section, $P_{1}$ : load, $P_{2}$ : pulling force, $P_{3}$ : motion initial caused by acceleration, $P_{4}$ : downward pulling force, $F$ : frictional force, $v$ : velocity in direction of belt motion, $d F_{1}: P_{1}$ side friction distribution in curve, $d F_{2}: P_{2}$ side friction distribution in curve.

net or rope began to slip on the belt, the maximum load tension was obtained, and the value was taken with a recorder. The static frictional force can thus be measured. As shown in Fig. 3, both pulling force $\boldsymbol{P}_{2}$ and load $\boldsymbol{P}_{1}$ act along the tangential direction of a spiral of the applied force system with frictional force. For the different pulling forces, a series of load data was obtained, hence the static frictional force generated could be measured and the frictional coefficient could be determined.

\section{Determination of Effect of Spiral Angle on Frictional} Force at Belt Velocity of Zero

By changing the contacting spiral angle between the synchronous belts and the net or the rope with different pulling forces, the tension in the net or rope at the loading end was measured by the tension meter cells. In the experiments, the spiral angles were set at $0,2.5,5.0,7.5,10.0$ and $12.5^{\circ}$, and the pulling forces were set at $70,105,165$ and 275 in $\mathrm{kgw}$.

Determination of Effect of Velocity on Frictional Force at Different Belt Velocities

In order to prevent deformation of the net or rope due to belt motion, an additional rigid profiling follower was set between two tension meter cells. The follower main- tains the spiral angle and the shape of the net or rope to keep normal running conditions. In the experiments, after the pulling force and predetermined load were applied, the belts started moving. When the net or rope began to slip on the belts together with the follower, the two cells in Fig. 2 detected the actual force and load at the same time. For each pulling force, by changing the velocity of the synchronous belts, the variation in frictional force for each velocity change was recorded. Based on the recorded data, the value of the pulling force and load was obtained and the effect of velocity on the frictional force could be analyzed.

During the experiments, a video camera was used to take films for image analysis. For each testing process, three-dimensional space pictures were taken to determine the parameters for the testing conditions such as magnitude of the spiral angle, arch length of the curved surface of net or rope, arch height, etc. The recorded pictures were resolved in partition-to-partition with an image analyzer (PIAS, model III) by the operation of intermittent circulation. The picture-taking illumination intensities were set at $280 \mathrm{l} x$ and $1,950 \mathrm{l} x$, and the image threshold values were set at 40 and 50. Through the picture analysis, the shape of the contact section between the synchronous belts and the net or rope was obtained and the size could be determined. Combining the front picture with the side picture by resolution analysis, the positional size for all the testing sections or parts could be measured.

\section{Results and Discussion}

\section{Frictional Force and Coefficient}

During the experiments, at each of six experimental sites, spiral angles from $0^{\circ}$ to $12.5^{\circ}$ were used and each of the six spiral angles corresponded to four pulling forces which ranged in value from 70 to $275 \mathrm{kgw}$. A group of four maximum loads was observed and recorded. For the six groups of recorded data, the relationship between the pulling force $P_{2}$ and load $P_{1}$ was as shown in Fig. 4 for the net and rope experiments respectively. By treating the recorded data with regression analysis, the relationship between $P_{1}$ and $P_{2}$ is given by the following equation:

$$
P_{1}=K \cdot P_{2}
$$

The regression coefficient $K$ and the correlation coefficient $r$ are shown in Table 1 .

In both the net and rope experiments, the relationship between the two end tensions $P_{1}$ and $P_{2}$ is clearly linear. Based on the measured data and the image analyzer, the working geometric shape between the synchronous belts and the net or rope was found to be a curve for any crosssection as shown in Fig. 3. Also, the relationship between pulling force $P_{2}$ and load $P_{1}$ was found to agree with Euler's equation for winding the net or rope on a circular roller. ${ }^{2-4)}$ The equation is

$$
P_{1}=P_{2} \mathrm{e}^{2 \mu \alpha}
$$

where $\alpha$ is the contact angle between the synchronous belts and the net or rope, $\mu$ is the frictional coefficient of the synchronous belts and the net or rope, and $\mathrm{e}$ is the base of the natural logarithm.

By applying Eq. (1) to the recorded data and performing 


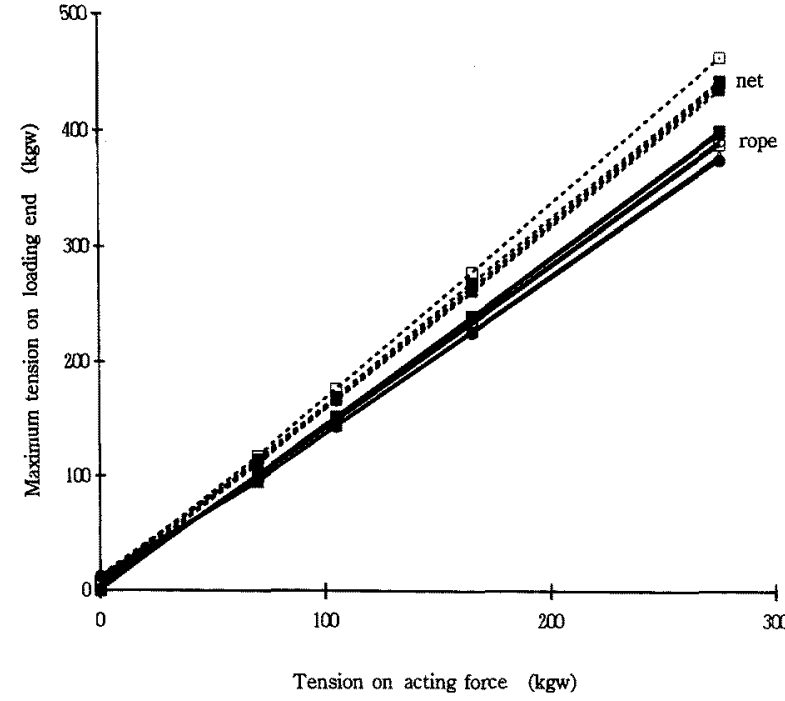

Fig. 4. Relationship between pulling force and load for different spiral angles for net or rope in static frictional experiments.

Table 1. The regression coefficient and correction coefficient of net and rope in static frictional experiments

\begin{tabular}{cccccc}
\hline & \multicolumn{2}{c}{$\begin{array}{c}\text { Regression } \\
\text { coefficient } \\
\begin{array}{c}\text { Spiral angle } \\
\beta\left({ }^{\circ}\right)\end{array}\end{array}$} & & & \multicolumn{2}{c}{$\begin{array}{c}\text { Correction } \\
\text { coefficient } \\
r\end{array}$} \\
\cline { 2 - 3 } \cline { 5 - 6 } & net & rope & & net & rope \\
\hline 0 & 1.63 & 1.46 & & 0.996 & 0.998 \\
2.5 & 1.59 & 1.45 & & 0.996 & 0.998 \\
5.0 & 1.61 & 1.43 & & 0.998 & 0.998 \\
7.5 & 1.69 & 1.42 & & 0.999 & 0.998 \\
10.0 & 1.60 & 1.38 & & 0.998 & 0.998 \\
12.5 & 1.60 & 1.37 & & 0.998 & 0.998 \\
\hline
\end{tabular}

theoretical calculations, it was found that when the contact angle $\alpha$ and frictional coefficient $\mu$ were a certain value, the load $P_{1}$ was a linear function of the pulling force $P_{2}$. From Eqs. (1) and (2), the frictional force can be analyzed. As shown in Fig. 3, the pulling force $P_{2}$ was applied by the outgoing end of the net or rope. When the load $P_{1}$ was gradually increased by the incoming end, the net or rope tended to move in the direction of application of the load.

In order to resist this tendency, opposing the acting direction, frictional force $\mathrm{F}$ was created between the synchronous belts and the net or rope. This frictional force is distributed along the contact curved surface of the synchronous belts and the net or rope as indicated by $F_{1}$ and $F_{2}$ in Fig. 3 and has two components of frictional force, $F_{n}$ and $F_{t} . F_{t}$ acts tangentially to the curve and $F_{n}$ in the direction of motion of the synchronous belts. These frictional forces are obtained from the following equations because the acting forces balance for any little length of the net or rope as shown in Fig. 3:

$$
\left.\begin{array}{l}
d F_{t}=d P \\
d F_{n}=\sin \beta \cdot d P \\
d F=\left(\sqrt{1+2 \cos \beta \cdot \sin \beta+\sin ^{2} \beta}\right) d P
\end{array}\right\}
$$

According to Eqs. (1) and (2), the relationship between the regression coefficient $K$ and frictional coefficient $\mu$ can be expressed as

$$
\mu=\frac{1}{2 \alpha} \ln K
$$

From Eqs. (1), (2) and (3), for all contact parts of the belts and net or rope, the frictional force is calculated by the following equation:

$$
\left.\begin{array}{l}
F_{t}=P_{2}\left(\mathrm{e}^{2 \mu \alpha}-1\right) \\
F_{n}=P_{2}\left(\mathrm{e}^{2 \mu \alpha}-1\right) \sin \beta \\
F=P_{2}\left(\mathrm{e}^{2 \mu \alpha}-1\right) \sqrt{1+2 \cos \beta \cdot \sin \beta+\sin ^{2} \beta}
\end{array}\right\}
$$

In order to determine the contact angle $\alpha$, without the influence of the spiral angle used in the analysis for calculating the frictional coefficient with the experimental data, the spiral angle was set to be equal to $0^{\circ}$. As described above, during the experiments the geometric shape of the contacting surfaces between the synchronous belts and the net or rope was found to be a curve through the whole section. Figure 3d shows a part of a section of such a curve with radius $r$ and circle center $O$. Based on image analysis and the measured data, the arch length $L$, the arch height $h$, and the width $b$ between belts can be obtained. From $L$ and $h$, the radius $r$ and the contact angle $\alpha$ can be calculated from the following equations:

$$
\begin{aligned}
& r=\frac{1}{2}\left(\frac{L^{2}}{4 h}+h\right) \\
& \alpha=\sin ^{-1} \frac{L / 2}{r}-\sin ^{-1} \frac{b / 2}{r}
\end{aligned}
$$

Table 2 shows the relationship between the geometrical position sizes of all the parts subjected to the different loads and the conditions carried out during the experiments.

From Eq. (4) and Tables 1 and 2, the average frictional coefficients between the synchronous belts and the net $\mu_{\mathrm{n}}$ and the rope $\mu_{\mathrm{r}}$ were calculated to be 0.78 and 0.70 , respectively.

The maximum frictional forces between the synchronous belts and the net or rope can be evaluated by combining Eq. (4) with the experimental data of frictional coefficient. Although the frictional coefficient changes with the material and the shape of the rope or net, the equation relating load $P_{1}$ and pulling force $P_{2}$ should be constant. Thus, as long as the frictional coefficients are determined by experimentation, the calculated frictional force can be used directly in the design of the pulling force and load for the hauling implements.

\section{Influence of Spiral Angle on Frictional Force}

From Fig. 4 and Table 1, the variation of the regression coefficient $K$ corresponding to a change of the spiral angle $\beta$ can be evaluated as follows. From the net experiments, the variation of the regression coefficient with a change in the spiral angle did not have any obvious effect on the frictional force. This was because the acting force and frictional force were distributed along the curved surface of the net or rope, and the spiral angle changing from $0^{\circ}$ to $12.5^{\circ}$ was in the range of the curved surface width. Thus, the change in the spiral angle was not large enough to displace 
Table 2. Geometric position size in net and rope experiments and the frictional coefficient

\begin{tabular}{|c|c|c|c|c|c|c|c|c|c|c|}
\hline \multirow{2}{*}{$\begin{array}{c}\text { Pulling } \\
\text { force }(\mathrm{kgw})\end{array}$} & \multicolumn{2}{|c|}{$\begin{array}{l}\text { Arch length } \\
L(\mathrm{~mm})\end{array}$} & \multicolumn{2}{|c|}{$\begin{array}{c}\text { Arch height } \\
h(\mathrm{~mm})\end{array}$} & \multicolumn{2}{|c|}{$\begin{array}{l}\text { Arch radius } \\
r(\mathrm{~mm})\end{array}$} & \multicolumn{2}{|c|}{$\begin{array}{c}\text { Contact angle } \\
2 \alpha(\mathrm{rad})\end{array}$} & \multicolumn{2}{|c|}{$\begin{array}{c}\text { Frictional coefficient } \\
\mu_{\mathrm{n}}\end{array}$} \\
\hline & net & rope & net & rope & net & rope & net & rope & net & rope \\
\hline 70 & 232 & 214 & 19.5 & 16.0 & 355 & 366 & 0.62 & 0.54 & 0.79 & 0.70 \\
\hline 105 & 232 & 214 & 19.5 & 16.0 & 355 & 366 & 0.62 & 0.54 & 0.79 & 0.70 \\
\hline 165 & 227 & 210 & 19.5 & 15.5 & 340 & 363 & 0.62 & 0.53 & 0.79 & 0.71 \\
\hline 275 & 223 & 207 & 19.0 & 15.5 & 336 & 354 & 0.62 & 0.54 & 0.78 & 0.70 \\
\hline
\end{tabular}

spiral angle $\beta=0^{\circ}$, moving up velocity $v=0$, correlation coefficient $K=1.63$

the forces from their lines reflected in the regression coefficient $K$. However, the variation of the spiral angle had some effect on the frictional force. This was confirmed by the data of the rope experiments (Fig. 4 and Table 1). In the case of the rope experiments, the applied force and frictional force acted along the curved surface of the curve for the main force acting on the net or rope as shown in Fig. 5. When the spiral angle is $0^{\circ}$, the circular $\operatorname{arch} \widehat{A B}$ of the curve for the main acting force becomes the contact angle $\alpha$ and the center of the circular arch is $O$. While if the spiral angle is $\beta$, the spiral circular arch $\widehat{A C}$ becomes the contact line of the equivalent contact angle $\alpha^{\prime}$ and the center of the circular arch is $O^{\prime}$. From the equation for calculating arch length in space, the length of $\overparen{A C}$, that is $l_{\overparen{A C}}$, is given by the following equation:

$$
l_{\overparen{A C}}=r \cdot \alpha \cdot \sec \beta
$$

For any position, the curvature radius $\rho$ of $\widehat{A C}$ of the spiral line is given by

$$
\rho=r\left(1+\tan ^{2} \beta\right)
$$

From this, the equivalent contact angle $\alpha^{\prime}$ can be obtained as follows:

$$
\alpha^{\prime}=\frac{l_{A C}}{\rho}=\alpha \cdot \cos \beta
$$

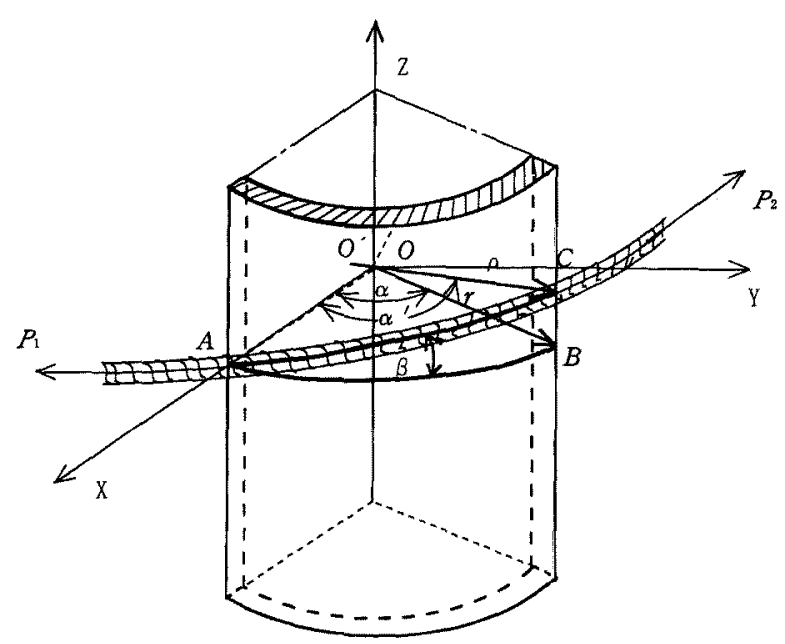

Fig. 5. Position of belt or rope in experiments.

$\alpha$ : contact angle, $\alpha^{\prime}$ : contact angle of spiral curve, $\beta$ : spiral angle, $r$ : radius of circular arch, $\rho$ : curvature radius of spiral line, $O$ : center of circle, $O^{\prime}$ : acting point $C$ of curve center of contact line.
When the spiral angle $\beta$ is not equal to $0^{\circ}$, Eq. (3) can be used to calculate the frictional force, if only the contact angle $\alpha$ is set equal to $\alpha^{\prime}$. According to Eq. (8), a decrease in $\alpha^{\prime}$ corresponds to an increase in the spiral angle $\beta$, which reduces the frictional force based on Eq. (5). For a small spiral angle $\beta$, small pulling force $P_{2}$, and rougher frictional contact surface such as in the net experiments, the influence on the frictional force is not significant and can be ignored in the calculation of the frictional force. However, as the pulling force $P_{2}$ and the spiral angle $\beta$ become larger, the influence is significant and the equivalent contact angle $\alpha^{\prime}$ of the spiral curve should be used instead of the contact angle $\alpha$ in calculating the frictional force in Eq. (5). To increase the frictional force, the spiral angle should be set as small as possible in the design of the net hauler or rope winch.

\section{Infiuence of Velocity on Frictional Force}

For uniform motion, the experimental data showed that increasing the moving velocity of the synchronous belts did not have any obvious effect or influence on the hauling rate of the load. Table 3 shows the various relationships among the different pulling forces, different constant velocities and the running loads at which the belts can move up when the spiral angle $\beta$ is $0^{\circ}$. By regression analysis on the data in Table 3 , the regression coefficients of the net and rope experiments ranged from 1.62 to 1.63 and 1.43 to 1.46 , respectively. Compared with the data of the static frictional experiment in Table 1 , the regression coefficients were found to be approximately constant.

This implies that for uniform operation, the contact relationship between the synchronous belts and the net or rope was static. Hence, the frictional force for the above case is static friction.

For normal running loads, the connection between the synchronous belts and the net or rope keeps the net or rope static relative to the synchronous belts because of the friction. In the experiments for a spiral angle of $0^{\circ}$, an abrupt change in the velocity, such as a sudden stop or jerk, did not have any significant influence on the maximum load at which the belts can move up. This can be explained by analyzing the forces in such an action. As shown in Fig. 3 , for a spiral angle $\beta$ of $0^{\circ}$, the pulling force $P_{2}$, load $P_{1}$, and frictional force $F$ are perpendicular to the direction of belt motion. In the direction parallel to the motion of the belt, no force acts.

However, if the spiral angle $\beta$ is not equal to $0^{\circ}$, over ac celeration leads to a reduction in the maximum load. Table 4 shows the data for the net experiments for a spiral an- 
Table 3. Relationship of moving up velocity and load in net and rope experiments

\begin{tabular}{|c|c|c|c|c|c|}
\hline \multicolumn{2}{|c|}{$\begin{array}{l}\text { Moving up velocity } \\
(\mathrm{m} / \mathrm{min})\end{array}$} & 12 & 14 & 17 & 19 \\
\hline \multicolumn{2}{|c|}{ Pulling force $(\mathrm{kgw})$} & \multicolumn{4}{|c|}{ Normal load (kgw) } \\
\hline \multirow{4}{*}{ Net } & 50.0 & 82.2 & 80.5 & 82.3 & 80.2 \\
\hline & 80.0 & 131.0 & 127.0 & 137.0 & 130.0 \\
\hline & 156.0 & 259.7 & 268.0 & 256.0 & 259.0 \\
\hline & 198.0 & 322.0 & 328.0 & 323.0 & 325.0 \\
\hline \multirow{4}{*}{ Rope } & 56.0 & 87.7 & 83.5 & 86.7 & 85.2 \\
\hline & 85.0 & 142.5 & 141.7 & 143.5 & 139.0 \\
\hline & 130.0 & 204.0 & 200.5 & 200.2 & 207.5 \\
\hline & 185.0 & 277.7 & 276.0 & 269.2 & 271.2 \\
\hline
\end{tabular}

Spiral angle $\beta=0^{\circ}$

Table 4. Relationship between maximum load and starting velocity in 5 second initial time with spiral angle $12.5^{\circ}$ for net experiments at pulling force of $50 \mathrm{kgw}$

\begin{tabular}{lrrrr}
\hline \hline Initial velocity (m/s) & 1.0 & 2.5 & 7.0 & 11.0 \\
Maximum load (kgw) & 79.0 & 75.7 & 70.0 & 65.2 \\
\hline
\end{tabular}

gle of $12.5^{\circ}$ and time during starting of 5 seconds. The maximum load at which the synchronous belts could move up decreased with increasing velocity. The effect of changing velocity on the frictional force which corresponded to the spiral angle of $12.5^{\circ}$ was clear. This is because when the spiral angle $\beta$ is not equal to $0^{\circ}$, a change in velocity results in acceleration, which tends to produce an inertia $d P_{3}$. The components of inertia $d P_{3}$ become part of the downward pulling force, which act in the opposite direction to the motion of the belts. As shown Fig. 3, in addition to the component $d P$, the downward pulling force $P_{4}$ can be calculated from

$$
\left.\begin{array}{l}
d P_{4}=\left(d P_{3}+d P\right) \sin \beta \\
P_{4}=\left(P_{1}-P_{2}\right)\left(\frac{a}{g}+1\right) \sin \beta
\end{array}\right\}
$$

where $P=m \cdot g ; P_{3}=m \cdot a, m$ is the mass of equivalent load, $a$ is the acceleration caused by the change in velocity, and $g$ is the acceleration of gravity.

For different velocity profiles, such as stopping or accelerating suddenly, a different inertia will be produced. If the downward pulling force $P_{4}$ reaches the value of the opposite frictional force component $F_{\mathrm{n}}$, the net or rope will begin to slip on the belts. That is:

$$
P_{4}>F_{\mathrm{n}}=P_{2}\left(\mathrm{e}^{2 \mu \alpha}-1\right) \sin \beta
$$

Thus, there is a change in the type of friction from a static friction to a kinetic one. Hence, the frictional force $F_{\mathrm{n}}$ will be greatly reduced. From Eqs. (9) and (10), for reducing the downward pulling force $P_{4}$, the load $P_{1}$ must be decreased or the pulling force $P_{2}$ must be increased. This is why the maximum load decreases during the starting operation with increasing velocity if the pulling force is a certain value. For uniform motion, there is no change in the frictional force especially for the moving up velocity of the belts. However, sudden changes in the velocity of the belts will produce additional loads which decrease the actual frictional force.

Through friction experiments and analysis of the experimental data, we concluded that it is possible for the synchronous belts to be used as transporting elements to move up the net or rope by utilizing friction in fishing machines. In fishing operations, the cross section of the belts, in which the main forces acted, was found to have a curved surface due to deformation. The tension relationship between the incoming end and outgoing end of the net or rope was linear when the contact angle of the synchronous belts with the rope or net was constant. However, the experiments were carried out for designing net haulers, and the values of the frictional coefficient might differ from those of accurate physical experiments. The data of the experiments and the values of regression analysis indicated the actual relationship of forces acting on the net and rope hauler and could be used in Euler's equation for the frictional calculation of pulling force and load. The spiral angle, which was formed as a result of the connection between the net or rope and the synchronous belts, could cause a decrease in the equivalent contact angle and consequently reduce the frictional force. Hence, the spiral angle should be smaller in order to increase the frictional force. Under normal working conditions, the moving up velocity of the synchronous belts did not have any significant effect on the frictional force. However, abrupt fluctuations of the velocity could create an extra inertia load which could decrease the actual frictional force.

By considering the above factors, we can use the experimental data and equations to determine the pulling force, the load, the frictional force and the geometric size in the design of purse siene net haulers and other fishing equipment.

\section{References}

1) N. Yamashita: Mechanical studies on the friction rope-net hauler. Bull. Fac. Fish. Hokkaido Univ., 33, 126-134 (1982).

2) H. Kristjonsson: Modern Fishing Gear of the World, Fishing News Press (book) Ltd., London, England, 1971, pp. 158-298.

3) The Japanese Society of Fisheries: Fisheries Books (Fishing Series), Press Department of Japanese Society of Fisheries, Tokyo, 1956, pp. 222-225.

4) C. Guangbin and S. Yada: Theoretical Analysis on New Mechanism of Purse Seine Net Hauling Vertical Drum. La mer, 33, 203207, (1995).

5) The Japanese Society of Mechanical Engineers: Machine Elements and Tribology. JSME Mechanical Engineers' Handbook (B1, Applications series), Tokyo, 1985, pp. 184-185.

6) T. Koyama: A Study on Strength of Toothed Belt. Transaction of the Japan Society of Mechanical Engineers (Series C), 399, 12601269 (1979).

7) M. Kagotani: A Study on Transmission Characteristics of Toothed Belt Drives (Series Reports). Transaction of the Japan Society of Mechanical Engineers (Series C), 419, 891-901 (1981).

8) M. Kagotani: A Study on Transmission Characteristics of Toothed Belt Drives (Series Reports). Transaction of the Japan Society of Mechanical Engineers (Series C), 429, 700-708 (1982).

9) M. Kagotani: A Study on Transmission Characteristics of Toothed Belt Drives (Series Reports). Transaction of the Japan Society of Mechanical Engineers (Series C), 451, 529-536 (1984).

10) M. Kagotani: A Study on Transmission Characteristics of Toothed Belt Drives (Series Reports). Transaction of the Japan Society of Mechanical Engineers (Series C), 466, 1354-1361 (1985). 